\title{
Editorial
}

\section{A NEW CLASSIFICATION FOR KERATOCONUS: AN ESSENTIAL STEP FORWARD IN TREATING THE DISEASE}

Keratoconus is described, typically, as a noninflammatory condition that resolves in corneal thinning in central paracentral areas of affected corneas. Whether there is or not an inflammatory process involved in keratoconus is at this moment under debate. ${ }^{1}$ What is not debatable is that, as the condition progresses, changes in the corneal regularity appear with the induction of first, high levels of regular and then irregular astigmatism with the corresponding degradation of the visual function.

The main complaint of keratoconus patients coming to our offices is visual loss. The severity of the keratoconus process concerning the progressive degradation of the corneal tissue, ending in some terminal cases to scarring and hydrops, is happening along a process in which visual loss is the main player in the patient's symptoms and concerns. The reason why the keratoconus patient accepts treatment is to gain, to restore the visual loss caused by the keratoconus. Our therapeutic alternatives also target this aim. Solutions, such as the adaptation of a therapeutic correcting contact lens which compensates part of the corneal irregularity, intracorneal ring segments and collagen cross-linking aim to achieve an improvement in best corrected and uncorrected visual acuity, something that does not always happen. Ultimately, modern techniques of corneal grafting are indicated with the purpose of improving the vision in these disabled eyes.

However, in spite of such strong clinical evidence, the deterioration of vision, being the main symptom of keratoconus, has never been considered to order the classification of the disease. Most of the available classifications of keratoconus have been established so far based on pachymetry, keratometry, corneal topography or sophisticated methods of measurement of corneal biomechanics. ${ }^{2,3}$ None of them have been considering vision, especially best corrected visual acuity, as the main issue involved in grading the disease.

Recently, we have proposed a classification which grades keratoconus based on the visual performance of the eye affected by the disease. ${ }^{4}$ We have demonstrated that this best corrected visual acuity decay is associated to a progressive deterioration in other parameters which involve the keratometry, corneal higher order aberrations, biomechanical analysis, such as corneal hysteresis and corneal resistance factors or the difference between these and, lastly but less important, pachymetry.

In other diseases, such as kidney filtration problems, heart disease, muscular skeletal abnormalities and many others, such as Alzheimer and other neurological diseases, the function which is affected by the disease is the main issue involved in the gradation of the illness. Why not do that in keratoconus? To know the disease better leads to better grading and to a better classification of a disease leads to a better indication of the therapeutic modalities available for the purpose. Like in tumor disease or heart function, the way to go in therapeutics is parallel to the degradation of the function. It is exactly like in keratoconus. Early stages, such as forme fruste or initial keratoconus, can be just observed without any intervention being necessary, as visual function is almost not degraded. Moderate stages may justify more invasive methods, such as collagen cross-linking or intracorneal ring segments or both associated, in which such corneoplasty methods (modifying the shape of the cornea), might improve the optical performance and, as a consequence, the visual function. Moderately severe cases might be less affected than the former by variables which can lead to a decrease in the vision of the patient in spite of the therapeutic modality use. Very advanced cases can be treated only by replacing the affected organ, i.e. by corneal transplantation. The clinical correlation between the grading of the disease and the therapeutic option and its outcomes has been also recently demonstrated in a recent publication of our group ${ }^{5}$ in which it has been demonstrated that early and moderate stages of keratoconus are not always positively affected by the use of intracorneal ring segments, which, on the contrary, are much more affected in advanced stages of the disease.

To know is to understand. To understand a disease is to treat it. The efficacy of the different therapeutic modalities will make us better doctors with our patients. Emerging therapeutic modalities, still under experimental evaluation, might find indications depending on the stage of the disease. In summary, we consider that the classification of keratoconus based on the visual degradation, i.e. decreasing best corrected visual acuity, related to the changes in other parameters is no doubt a huge step forward in the understanding and the investigation of the disease and also opens the door to a better and new therapeutic indication of the different conservative and invasive therapeutic alternatives existing today. 


\section{REFERENCES}

1. Balasubramanian SA, Pye DC, Willcox MD. Are proteinases the reason for keratoconus? Curr Eye Res 2010 Mar;35(3):185-191.

2. Lovisolo CF, Calossi A, Ottone AC. Intrastromal inserts in keratoconus and ectatic corneal conditions. In: Lovisolo CF, Fleming JF, Pesando PM, editors. Intrastromal corneal ring segments. Canelli AT, Italy: Fabiano Editore; 2000. p 95-163.

3. Alió JL, Shabayek MH. Corneal higher order aberrations: a method to grade keratoconus. J Refract Surg 2006;22:539-545.

4. Vega-Estrada A, Alio JL, Brenner LF, et al. Outcome analysis of intracorneal ring segments for the treatment of keratoconus based on visual, refractive, and aberrometric impairment. Am J Ophthalmol 2013;155:575-584.

5. Alió JL, Piñero DP, Alesón A, et al. Keratoconus-integrated characterization considering anterior corneal aberrations, internal astigmatism, and corneal biomechanics. J Cataract Refract Surg 2011;37:552-568.

Jorge L Alio MD, PhD, FEBO

Professor and Chairman of Ophthalmology Miguel Hernandez University, Alicante, Spain

President, Vissum Corporation, Spain 